\title{
Scenario for the Origin of Matter (According to the Theory of Relation)
}

\author{
Russell Bagdoo \\ Saint-Bruno-de-Montarville, Quebec, Canada \\ Email: rbagdoo@gmail.com,rbagdoo@yahoo.ca
}

How to cite this paper: Bagdoo, R. (2019) Scenario for the Origin of Matter (According to the Theory of Relation). Journal of Modern Physics, 10, 163-175.

https://doi.org/10.4236/jmp.2019.102013

Received: February 20, 2019

Accepted: February 24, 2019

Published: February 27, 2019

Copyright $\odot 2019$ by author(s) and Scientific Research Publishing Inc. This work is licensed under the Creative Commons Attribution International License (CC BY 4.0).

http://creativecommons.org/licenses/by/4.0/

\begin{abstract}
Where did matter in the universe come from? Where does the mass of matter come from? Particle physicists have used the knowledge acquired in matter and space to imagine a standard scenario to provide satisfactory answers to these major questions. The dominant thought to explain the absence of antimatter in nature is that we had an initially symmetrical universe made of matter and antimatter and that a dissymmetry would have sufficed for more matter having constituted our world than antimatter. This dissymmetry would arise from an anomaly in the number of neutrinos resulting from nuclear reactions which suggest the existence of a new type of titanic neutrino who would exceed the possibilities of the standard model and would justify the absence of antimatter in the macrocosm. We believe that another scenario could better explain why we observe only matter. It involves the validation of the negative energy solution of the Dirac equation, itself derived from the Einstein energy equation. The theory of Relation describes a negative energy ocean with the creation of real particle/antiparticle pairs. The origin of the masses of the particles would come from this ocean. A physical mechanism would allow their separation in the opposite direction and, therefore, the matter would be enriched at the expense of the ocean. The matter would be favored without resorting to negation or annihilation of negative energy, without the need for a CP (the behavioral difference between particle and antiparticle) violation that would be responsible for matter/antimatter asymmetry in the universe. And without the savior contribution of an undetectable obese neutrino: his search appears to us more a desperate act towards an "ultra-massive catastrophe" than a real effort to try to discover what really happened.
\end{abstract}

\section{Keywords}

Matter and Antimatter, Sterile Neutrino, Ocean of Negative Energy, Theory of Relation, Pair of Real Particles, Principle of Compensation 


\section{Introduction}

The standard model of the big bang makes it possible to reconstruct the history of the cosmos in large part, in good agreement with the astronomical observations, until the first fractions of a second that followed the zero-time. In spite of this, we have not succeeded in combining the three fields, electromagnetic, electro-weak and strong into a single one at very high interaction energies, and we are unable to reconcile the requirements of quantum mechanics with those of general relativity to quantify the gravitational field. This has not prevented the theoreticians of particle physics, astrophysics, and cosmology from engaging in the craziest speculations to answer some of the great questions that aspire to explain our universe.

One of the questions that seem to have found a satisfactory answer concerns particle physicists: How did matter appear? The consensus is reached on the idea that in the moments following the initial moment of the big bang, when the universe is in a neutral matter state of photons for the most part and neutrinos, this neutral matter will transform and separate into matter and antimatter which will re-annihilate, etc., up to the present stage. The question of whether at the beginning the universe was completely neutral, which would imply that there is now as much antimatter as matter, seems to be discarded since one does not find constituted antimatter. The current dominant idea is that of the Soviet physicist Andrei Sakharov: the universe was slightly biased on the matter side, which would explain its predominance at the present time [1] [2].

To corroborate the idea of Sakharov, physicists rely on certain physics experiments, some disintegrations which generate a dissymmetry coupled with the particle/antiparticle symmetry. It is believed that a small dissymmetry, similar to that of kaons which decompose differently from their antiparticle, would suffice to leave a tiny excess of baryons with respect to the antibaryons. But we now prefer experiments with leptons. Thus, the first results of the T2K experiment carried out since 2011 in Tokai, Japan, indicate that a very slight imbalance may have appeared during the disintegration of certain particles: heavy neutrinos. This reaction gives birth to leptons (electron, muon, tau) or antileptons, but not in equal proportions: for 100,000 antileptons, 100,001 leptons would be formed. It suggests that during the big bang, slightly more matter was created than antimatter.

In our view, the thesis of a small violation of particle/antiparticle symmetry at the first moments of the universe is not a theoretical necessity. The argument that this difference will prove to be crucial to demonstrate that after the appearance of matter and antimatter at about $10^{-30}$ seconds, obese, ultra-massive neutrinos would have broken the equilibrium of the cosmos, seems to us unfounded and desperate.

The intention of this article is to propose an alternative to the direction taken to answer the question How did matter appear? In Section 2, we show that Sakharov's idea of an original dissymmetry that would have privileged matter is biased and causes particle physics towards dead-end directions. Any solution of 
negative energy is treated as non-physical, despite the fact that they are mathematically valid predictions of the Dirac equation. Several experiments have highlighted the concept of oscillation which surmises that neutrinos can change their flavor throughout their journey because they have a mass. It has been found that the number of antineutrinos produced by a reactor is lower than theoretically expected, which evokes an original dissymmetry. Physicists speculate the existence of heavy neutrinos at the exit of the reactors which would accredit the process of oscillation, could constitute the dark matter and would explain the absence of the antimatter of our universe. We think they are putting their finger in the eye too quickly. In Section 3, we show that Dirac's negative energy solution, banned from physics, is a reality. But it is a question of a virtual reality in which the sea of negative energy materializes above the surface of the pairs of particle/antiparticle which annihilate immediately. We present an alternative in Section 4, namely the solution of the negative energy of the theory of Relation. Throughout the expansion, the highly intense electromagnetic field of the ocean spontaneously converts energy into particles/antiparticles, both for matter and space. A separation mechanism allows the creation of real particles of positive energy. A new variable $\left(\mathrm{M}_{\mathrm{vp}}^{2}\right)$, from the equation of the theory of Relation, gives mass to these particles.

\section{Origin of the Matter According to the Idea of Sakharov}

\subsection{Sakharov's Idea of a Dissymmetry at the Origin of Matter in the Universe}

Today, the consensus is that although there would have been an initially symmetrical universe made of matter and antimatter, there would still have been some dissymmetry in the particle laws, and this dissymmetry would have sufficed so that more particles remain than antiparticles, and this would explain why there would have been a small excess of particles which would have served to fabricate the cosmos we know [3].

This consensus starts from the idea of Sakharov of an original dissymmetry that would have advantaged matter. He posed the problem in the form of three conditions. The first-that the universe is in a state of violent thermal imbalance-is fulfilled by the standard cosmological model of the big bang. The second-that matter can be transformed into antimatter-is, also, already authorized by the standard model of particles. The third condition dives at the heart of the problem. To build a world filled with matter, the processes that transform matter into antimatter must violate the combination of two symmetries: the symmetry $\mathrm{C}$ (charge), which stipulates that a process remains unchanged if the charges of the particles involved are changed; and the symmetry P (parity), according to which if a transformation occurs in nature, then the transformation which would be the image in a mirror is just as possible. In the early 1960s, it was observed that some unstable particles, kaons, decompose slightly differently from their antiparticle. It was thought that this small violation of parti- 
cle/antiparticle destroyed the initial perfection of nature. It has been anticipated that a small similar dissymmetry between baryons with respect to the anti-baryons which would leave a tiny excess of baryons would prove that the universe is only made by baryons. So far no proton disintegration has ever been observed. The physicists then turned to the leptons, concentrating on the neutrinos. The T2K experiment (Tokkai to Kamioka) has been working to observe the transformation of a neutrino type, the muon neutrino, into an electron neutrino. They then compared with the transformation of muonic antineutrinos into electronic antineutrinos. After two years of measurements, they found too many neutrinos detected and not enough antineutrinos, proof of a violation of the CP symmetry [2].

This second anomaly is, for theorists, an indicator of a violation of symmetry between matter and antimatter. Such a violation goes against the current standard particle model and, moreover, has the theoretical consequence of sowing a disorder that invalidates its current formulation [4]. But it must be recognized, until today, neutrinos of the standard model do not agree with the data of the observation and do not allow, in particular, to explain the deficit of antineutrinos compared to the excess neutrinos. According to experts, these anomalies should be the manifestation of a particle still unknown. A particle which, on the one hand, is the result of the mutation of a standard neutrino, from which their disappearance, and which, on the other hand, is capable of transforming into a standard neutrino, what would explain the excesses observed. This particle would be a neutrino of a fourth type not yet detected. They called it "sterile neutrino" because it is not sensitive to any of the three basic interactions of the standard model. Not even to the weak nuclear force. It interacts only through gravitation (almost zero intensity at the particle scale and undetectable in the context of a microscopic physics experiment). They suspect it endowed with a state of right-handed helicity. Such a particle could not only explain how matter prevailed over antimatter but it could also be the original particle from which the mass of matter comes.

\subsection{The Original Particle from Which Comes the Mass of Matter}

Only a few years ago, the particle that confers its mass on all the others was the Higgs boson which represented the Brout-Englert-Higgs (BEH) field. It is an unstable particle that survives barely $10^{-22}$ seconds after its production. The boson is not observed since it disintegrates immediately by splitting into other particles which can be observed. In fact, it is only an excitation of the BEH field which can be compared to an ocean surface. To excite the ocean and produce waves, it is enough to supply energy, whether through wind, tidal power or an earthquake. We excite the ocean of BEH by supplying it with energy with particle accelerators. This excitation or wave is none other than the scalar boson of Higgs and it is manufactured exactly as is manufactured the antiparticle in the accelerators. 
Theorists had suggested that the universe was filled with an ocean of BEH. Other physicists have proved that this ocean (or field) exists by creating excitations of this field in the form of Higgs bosons, thus completing the standard particle model. In the end, most physicists are disappointed because there are no waves without water and no Higgs boson without BEH ocean. For them, conceptually, the incoherence of an ocean of negative energy (that it bears the name of Dirac, Higgs, or other) is monumental: it deals with an ocean that physically has no consistency, even if it holds some exotic properties; An ocean that connects two kinds of energy that ignore each other is very inconsistent. Detection of the boson of Higgs completes the standard model but to go beyond, towards an ocean of negative energy, is forbidden by the official Physics and excluded from the model [5].

In fact, the problem is circumvented from the moment we perpetuate the hypothesis that everything is only of positive energy. By deciding that the physical properties of particles and antiparticles are only positive energy, physicists impose ipso facto a radical dissymmetry that violates $\mathrm{CP}$ symmetry and annihilates the antimatter. For the hypothesis to continue to work, it is imperative to put in the closet the particle of God, that has become cumbersome and without future. Dethroned, it will quickly be replaced by the sterile neutrino, the fourth type. The new physics of neutrinos, with this invisible savior, should explode the standard model and entail vast cosmological consequences, such as making dark matter, justify the absence of antimatter in the universe.

\subsection{The Sterile Neutrino, the Perfect Phantom Particle that Succeeds the Higgs Boson}

According to specialists, the tiny relic of the disintegration of super heavy neutrinos and antineutrinos would have tipped the cosmic balance towards the matter. The imbalance of the cosmos towards $10^{-30}$ seconds would have been transmitted to the quarks, then to the protons and neutrons until the great annihilation towards $10^{-4}$ seconds, destroying all the antiparticles to leave alone the grains of matter which will be structured in atoms, stars, planets. This undetectable particle would explain the failure counts in the neutrino flux measured for more than fifteen years near nuclear reactors. Detectors at the output of nuclear reactors have detected more neutrinos than antineutrinos. The number of antineutrinos measured is $7 \%$ lower than that theoretically expected. As if they had mysteriously disappeared. This flagrant anomaly would not only break the restrictive framework of the standard model of particle physics but would also have unbalanced the cosmos $10^{-27}$ seconds after the big bang, having thus given birth to all the matter of the universe and having been able to constitute dark matter, i.e., $85 \%$ of the mass of the natural world.

This scenario uses data from the $\mathrm{T} 2 \mathrm{~K}$ experiment that argue in favor of the $\mathrm{CP}$ symmetry violation, about 1000 times stronger than in processes involving quarks. Even with this asymmetry, these neutrinos do not make the weight to explain the predominance of matter in the universe. According to the models, 
the smallness of the masses of the three classic neutrinos can only be explained by means of a fourth type of neutrino, heavy as tens of billions of protons. A primordial version of the current neutrinos, that must have existed at the beginning of the creation. Theoreticians are obliged to introduce a titanic sterile neutrino, with a mass-energy of the order of $10^{9}$ teraelectronvolts $(\mathrm{TeV})$. However, such energy far exceeds the possibilities of describing the standard model, whose equations become crazy when they attempt to describe elementary phenomena involving energy greater than a few $\mathrm{TeV}$.

The tracking down to these obese neutrinos susceptible to solve the problem of the mass of their congeners is already launched. Current experiments use the oscillation phenomenon, valid for any type of neutrino, to flush them out. An alternation of appearances and disappearances of standard neutrinos, near nuclear power stations for several months, should suffice to conclude that the undetectable sterile neutrino is unmasked [2] [6].

\subsection{Neutrino with Neither Faith Nor Law}

In view of the experimental success of the discovery of the Higgs boson, it is expected that the sterile neutrino will not be a theorist's simple whim, but will be demonstrated in the next few years through experiments [7]. And yet could it be that the thought of the neutrino specialists was misled? Is the neutrino oscillations phenomenon, crowned by a Nobel Prize, merely a praise of error? Would not the building of twenty years of experience and data build upon a gigantic mistake?

Then if the sterile neutrino exists for nuclear reactors and acts on the electronic neutrinos, why would it not exist for the Sun, the ideal nuclear reactor which produces only electronic neutrinos? If the sterile neutrinos act on the electronic neutrinos of the Sun, before or at the photosphere exit, and we do not see why it would be otherwise, it would mean that the theory of the oscillation would be false.

It is known that two-thirds of the electron-type neutrinos produced by nuclear reactions in the core of the Sun are observed to change to muon- or tau-type neutrinos before reaching the Earth. The experience of the Sudbury Neutrino Observatory (SNO) would have demonstrated that a good part of the electronic neutrinos emitted by the Sun is transformed into muon and tau neutrinos along the way. The SNO detector, using heavy water, a substance allowing to detect the three flavors of neutrinos, could have verified that the sum of the three neutrino types corresponded to a number of electronic neutrinos produced by the Sun. The specific SNO measures would thus have demonstrated the ability of neutrinos to change from one type to another in transit to the Earth from the Sun, to "oscillate", proving by the very fact that they have finite masses. But would this demonstration be as well obvious with this new invisible parameter, the sterile neutrino?

With this fourth player, would the sum of the three neutrinos flavors corre- 
spond to an amount of electronic neutrinos produced by the Sun? It is known that the missing standard neutrinos are transformed on the way into another type and that such a transformation requires at least a great distance. If the sterile neutrino changes the identity of the neutrinos without any distance, it is that there are not many oscillations over great distances. So the theory of neutrino oscillation would be wobbly. Especially since the neutrinos from the supernovae 1987 A [8] were traveling at substantially the same speed as the photon, which would not have been the case if they had had a mass [9].

One would have the new parameter of the heavy neutrino for the very short distances which explains an unforeseen deficit and the oscillation of the mass states of the light neutrinos for the great distances which explains the total deficit. These two deficits are contradictory and one can apprehend an "ultra-massive catastrophe". Which reminds the "ultraviolet catastrophe" at the end of the 19th century concerning the radiation emitted by the bodies being heated; we had the Wien law which reported experimental observations for short wavelengths without being capable for long wavelengths, and Rayleigh's law for long wavelengths but which did not diverge for small wavelengths [10]. The neutrino oscillation hypothesis and the sterile neutrino hypothesis contradict each other. Which is false? What if both were false? What if the negative energy ocean existed? What if the antineutrinos were attracted by the ocean?

\section{Dirac's Negative Energy Solution}

\subsection{The Original Swindle}

At the outset, the thesis of an alleged symmetrical creation made of matter and antimatter, with an asymmetry in the laws concerning the particles that would have sufficed so that it remains a small excess of particles used to produce the universe, is not based on sound scientific evidence. It has been totally fabricated from an idea issued in 1967 by Sakharov. This idea goes beyond the hypothesis for practically all specialists. It takes on the dimension of a true scientific theory that explains the origin of matter. But it is also a form of scam. For the simple reason that we are talking here about a strictly positive energy solution framework and that physics has officially eliminated the negative energy solution. Which gives a speculative and unfounded character to the idea of an originally symmetrical universe, but in which a particular mechanism would have quickly preferred matter [11].

For those who might doubt it, it should be stressed that it is foolish to want to apply only the equations of Einstein's special relativity concerning positive energy at the moment when the age of the universe is equal to Planck's time. All physicists know that the energy-momentum-mass relationship of the Dirac equation $E^{2}=c^{2} p^{2}+m^{2} c^{4}$, which is always associated with Einstein's special relativity, has two roots. Those of positive energy and negative energy: $\pm E=\left(c^{2} p^{2}+\right.$ $\left.m^{2} c^{4}\right)^{1 / 2}$. The negative energy solution is that of Dirac sea. Under the pretext that it could not be observed directly, Heisenberg, Pauli, Jordan, and others, have ex- 
cluded all negative energy solutions from classical physics; it is, therefore, illegitimate to use negative energy solutions in a quantum period where there are only particles and antiparticles. How do we want the concept of matter, a notion which is the basis of our universe, has a meaning at the particle and atom level, whereas the deep concept of antimatter is eliminated as a theoretical chimera from the equations and laws of quantum mechanics [2] [12]?

It was the original swindle which ended in the total annihilation of the antimatter. The so practical causal dissymmetry is equivalent to the exclusion of the negative energy solution from the equations. And it is, in our opinion, because of this negation of a Dirac sea, that particle physicists no longer know what to do with the Higgs boson, and that the BEH field (which is the Dirac sea under a new name) is now becoming a skeleton in the closet of particle physics.

\subsection{Solution of Dirac Equation's Negative Energy}

To solve this thorny problem, we postulate that contrary to what is assumed by classical physics, negative energy actually exists as much as positive energy. In a universe of only positive energy, the mechanism that was chosen to transform matter into antimatter is that of Sakharov: the universe would have been originally symmetrical, but soon after the materialization of particles and antiparticles, a mechanism of disintegration before annihilation would have quickly chosen matter. The other processes originally asymmetric universe, an originally symmetric universe where particles and antiparticles separate to form galaxies and anti-galaxies, an originally symmetric universe favoring the antimatter were ruled out. Several physicists have already proclaimed the existence of the Dirac sea of negative energy.

\subsection{Creation of a Virtual Particle/Antiparticle Pair and Annihilation}

The equation of Dirac gives to a particle of mass $m$ the possibility of having negative as well as positive energies. Dirac interpreted the result assuming that all states of negative energy are occupied by unobservable electrons, forming an invisible "sea", the electrons of positive energy floating on this sea being the only observed ones. The hypothesis may seem ad hoc. However, Dirac added with the next argument: If a photon of energy $h v$ greater than $2 \mathrm{mc}^{2}$ interacts with an electron of the sea of negative energy $-\varepsilon_{0}\left(\varepsilon_{0}>0\right)$, he can communicate his energy and make it pass in a state of energy $h v-\varepsilon_{0}$; this electron is then observable. The two symmetrical solutions of this equation, one of positive energy and the other of negative energy, allowed him to postulate the existence of particles analogous to the electron but of opposite electrical charge. With Anderson who discovers the positron in 1932, appears the reality of the antimatter, as Dirac equation predicted, without physicists having discerned it. It will then be discovered that almost all the particles are matched to antiparticles [13] [14] [15].

Would we have created matter and electric charge from energy with the negative energy solution of Dirac equation? It is not believed because it has been as- 
sumed that there is now a "bubble" or "hole" in the sea.

\subsection{Dirac's Negative Energy Solution Unacceptable to Explain the Asymmetry between Matter and Antimatter}

The Dirac sea despite the constant annihilations and creations of "pairs", total transformations of mass in energy, of energy in mass in no way diminishes its level of water in favor of the ground (matter). The emersion of the ground, which leaves a hole in the water, is immediately harpooned by the sea which hates the decreasing water levels, no matter how high the waves. In other words, the electromagnetic energy $\left(E=m c^{2}\right)$ is turned into mass-energy of the particle and the antiparticle (which leaves only a hole in the sea because the antiparticle is only a mirror image) but the two are condemned to annihilate in a flash of energy to ensure the stability of the ocean. Despite their undeniable spectacular metamorphoses, these negative energy solutions remain virtual and are physically unacceptable to explain the asymmetry between matter and antimatter.

\section{Origin of Matter According to the Theory of Relation}

\subsection{Negative Energy Solution Would Involve the Creation of a Pair of Real Particles}

However, the ocean of the theory of Relation [16] [17] can explain why today the universe consists essentially of matter whereas matter and antimatter must have been produced in equal quantities after the big bang. It is worth remembering that according to the theory our universe has two structures: a structure of expansion with an expanding negative electromagnetic energy (identified or equivalent to dark energy, to $\mathrm{BEH}$ ocean or to the Dirac sea) and a structure of condensation that uses known matter and general relativity. There is cooperation, not competition, between these two antagonistic structures, and it is this cooperation that determines the nature of expansion. The structure of the expansion comes from a universe that has decomposed and has transferred its energy to the condensation structure of our universe that he gave birth to. The process would have started with a big bang (we assume a pre-existing quantum plenum) in the Planck era and would continue again. The compensation principle ensures that the negative energy of the structure of the expansion is transformed into positive energy of the structure of the condensation. The total energy density remains constant, although the two energy contributions respond to the expansion in a radically different way. The first, the energy density of the quantum "vacuum" of the structure of expansion, is progressively diluted, like that of any ordinary fluid housed in a volume that extends. It plays the role of a negative cosmological constant. It tends to decelerate the expansion and solves the problem of the vacuum catastrophe: The value of the energy of the vacuum today corresponds to the cosmological value observed, which is $10^{120}$ times smaller than that calculated by the quantum theory for the Planck era. The second, the density of the energy of the structure of the condensation, which comes 
from the lost energy of the quantum vacuum, increases and condenses progressively, like that of any ordinary fluid lodged in a volume that shrinks. This is how stars, galaxies, clusters of galaxies are formed.

In addition to this duality, note that the Dirac sea applied to the atom while the ocean of the theory of Relation concerns the atom and the vacuum of space, hence the term "ocean" instead of "sea". Before elaborating further, let's say that the asymmetry between matter and antimatter is mainly explained by the creation of a pair of real particles (leaving two holes in the ocean); the antiparticle annihilates itself by regaining its oceanic hole while the particle is added to the matter leaving an oceanic hole. All in all, the hypothesis of the sea of Dirac is beautiful, but defective to explain the asymmetry between matter and antimatter. The particles of this sea are virtual whereas they are a reality in the ocean of the theory of Relation, which makes all the difference. The Dirac sea hypothesis is beautiful, but defective in explaining the asymmetry between matter and antimatter, while that of the ocean of the theory of Relation is effective.

\subsection{Ocean of Negative Energy Seen by the Theory of Relation}

Since all forms of energy is equivalent to mass, it is logical to expect that electromagnetic energy can also be converted spontaneously into particles. This is precisely the deep meaning of our mathematical model of the universe. The quantum vacuum is polarized by the very intense electromagnetic field that prevails in the ocean of negative energy. Above this ocean, pairs are constantly created and destroyed. The very intense electromagnetic field of the ocean spontaneously converts energy into particles. For a brief moment, a particle and its antiparticle separate. There are then four possibilities: Process 1: the two partners meet and annihilate.

Process 2: the negative energy antiparticle is captured by the negative energy ocean and the negative energy particle materializes in the outer world. The latter becomes an antiparticle of positive energy.

Process 3: The negative energy particle is captured by the ocean and its partner (negative energy antiparticle) escapes to become a positive energy particle.

Process 4: Both partners plunge into the negative energy ocean.

Since matter predominates, we incline towards process 3 . The energy balance of the negative energy ocean of the theory of Relation shall be the following: by preferentially capturing particles of negative energy (the latter will encounter antiparticles of negative energy: they will annihilate each other in the ocean or they will materialize above) and by losing antiparticles of negative energy, the ocean spontaneously loses energy, hence mass. For an outside observer, lands of matter emerge on the horizon while the ocean evaporates by emitting radiation and particles. It is what explains the asymmetry between matter and antimatter.

\subsection{Creation of a Real Particle/Antiparticle Pair and Separation}

The ocean of the theory of Relation is filled with particles and antiparticles of all 
kinds. If a photon of energy $h v$ greater than $4 m c^{2}$ interacts with an electron and a positron of the ocean of negative energy $-\varepsilon_{0}\left(\varepsilon_{0}>0\right)$, it can communicate to them its energy and make them go in a state of energy $h v-\varepsilon_{0}$; This electron and this positron are then observable. There are now two "bubbles" or two "holes" in the ocean.

The holed ocean is equivalent to a full ocean and to two objects, one of positive energy $+\varepsilon_{\mathrm{o}}$ and of charge $+\mathrm{e}$ opposite to those of the electron, the other of positive energy $+\varepsilon_{\mathrm{o}}$ and of charge $-\mathrm{e}$ opposite to those of the positron. The initial photon disappeared and created a real electron and a real positron, that is, an electron with a positron hole and a positron with an electron hole. Four entities: two pairs of particle and antiparticle, a pair of positive energy over the ocean that can be observed and a pair of holes in the negative energy ocean.

The particularity of the mechanism would be this: a photon which has in itself its particle and its antiparticle makes cross a pair electron-positron of negative energy the barrier that separates the negative energy from the positive energy. The negative energy electron becomes a positron of positive energy while the positron of negative energy becomes the positive energy electron. There is then materialization of the electron-positron pair. Afterward, the electron and the positron separate each going in an opposite direction. The positron of positive energy will regain the negative energy ocean and will become again the negative energy electron. There will at that time annihilation equivalent to $2 \mathrm{mc}^{2}$. As for the positive energy electron, it will add $2 m c^{2}$ to the matter and leave a hole in the ocean that will have the image of an electron of negative energy.

The conservation of energy is preserved but it can be said that there is a creation of matter and electric charge from energy since there is manifestly a preponderance of matter on antimatter. There is currently no evidence that antimatter exists in the world in the form of atoms, like matter, in appreciable quantities. Antimatter is only observed in the form of isolated antiparticles, which are produced either by cosmic radiation or by large accelerators. Negative energy appears to be liquid cement destined to produce matter.

\subsection{How the Mass Comes to Particles}

This cement also gives masses to the particles. It is akin to the Higgs mechanism. In the article "The Equation of the Universe" [18], we have seen that the flat spatiotemporal ocean of special relativity merges with the ocean of Higgs, itself assimilated with the Dirac sea, amalgamated with the ether ocean (minimum vacuum energy). Under the Principle of Compensation of the theory of Relation, there is a continuous transformation of so-called "negative" energy into "positive" energy. The principle of Compensation says that the decrement of negative electromagnetic energy-mass during the expansion induces a proportional and opposite increment of the positive gravitational energy-mass [16].

According to the equation $\mathrm{ke}^{2}=\mathrm{M}_{\mathrm{vp}}^{2} \mathrm{t}_{\mathrm{o}} \mathrm{c}$ of the theory of Relation [here $\mathrm{M}_{\mathrm{op}}$ is the proton rest mass; $M_{o p}\left(1 /\left(1-v^{2} / c^{2}\right)^{1 / 2}\right)$ gives the relativized proton $M_{v p}$, i.e., the rest mass + the kinetic energy; $v=$ the estimated recessional velocity of the 
galaxies], or more precisely $\pm \mathrm{ke}^{2}= \pm\left[\mathrm{M}_{\mathrm{op}} /\left(1-\mathrm{v}^{2} / \mathrm{c}^{2}\right)^{1 / 2}\right]^{2} \mathrm{t}_{\mathrm{o}} \mathrm{c}$, since the particles come in pairs, each with a counterpart antiparticle, the term $\mathrm{M}_{\mathrm{vp}}^{2}$, or $\left[\mathrm{M}_{\mathrm{op}} /(1-\right.$ $\left.\left.\mathrm{v}^{2} / \mathrm{c}^{2}\right)^{1 / 2}\right]^{2}$, is a new fundamental variable in physics. Its value changes throughout the expansion. We suspect it to be the non-zero average value in the vacuum of the Higgs field. It would be the scalar field of the ocean of Higgs at the origin of the inertia of matter which measures the force that must be applied to an object to print it a given acceleration. It would follow a transformation of a space-time more and more flat into a space-time more and more locally curved.

Before being a boson that contains a particle and an antiparticle, $\mathrm{M}_{\mathrm{vp}}^{2}$ is a field that gives masses to the particles of the four forces. It blends in with the Higgs mechanism. Like him, he is as a mud in a field that sticks to the boots which thus become heavier. Similarly, particles-boots begin weighing.

\section{Conclusions}

Particle physicists assume the existence of an undetectable ultra-massive sterile neutrino which, not only would explain the anomalies in the neutrinos flux measured near nuclear reactors but which would have unbalanced the cosmos after the big bang, thereby giving birth to all matter in the universe, including dark matter [2] [6]. Excited, they hope that an alternation of appearances and disappearances of standard neutrinos near nuclear reactors for several months should suffice to conclude that the sterile neutrino is discovered. We think that the hypothesis of a fourth neutrino, very heavy, following the discovery of a deficit of antineutrinos, contributes more to invalidating the phenomenon of the oscillation of neutrinos than to fortify it. The contradiction is too blatant between the oscillation of standard neutrinos over long distances and the oscillation of the sterile neutrino with standard neutrinos over virtually non-existent distances.

Unlike neutrino physicists, we consider that such an alternation of neutrinos appearances and disappearances near nuclear power stations would mean a completely different phenomenon coming from a negative energy field. According to the theory of Relation, the origin of the masses of the particles would come from the ocean of negative energy. There would be a physical mechanism, likely beyond the standard model, at the origin of elementary particle masses. We have described it above: the creation of a pair of real particle/antiparticle and their separation in opposite direction, from which it follows that ground (matter) levels are rising while the ocean' levels are going down [7]. Energy power near the reactors would be better able to create neutrino-antineutrino pairs in favor of neutrinos. The natural tendency of antineutrinos would be to join the ocean of negative energy. One would not need a CP violation that would be responsible for the matter/antimatter asymmetry in the universe. It is ironic to think that Dirac's negative energy solution was perceived as non-existent. On the contrary, its relations with cosmology play in its favor and, more generally, it would be its origin. 


\section{Conflicts of Interest}

The author declares no conflicts of interest regarding the publication of this paper.

\section{References}

[1] Lévy-Leblond, J.-M. (1981) La matière aujourd'hui. Seuil-Point, 119, 136.

[2] Grousson, M. (2016) Science \& Vie, 1191, 48, 56-59.

[3] Schaeffer, R. (1986) Chaos et Cosmos, L’anti-matière. Éditions Le Mail, 111.

[4] Schatzman, E. (1989) L'expansion de l'Univers. Hachette, 60, 61.

[5] Gagnon, P. (2016) Who Cares about Particle Physics? Oxford University Press, Oxford, 67-68. https://doi.org/10.1093/acprof:oso/9780198783244.001.0001

[6] Grousson, M. (2012) Science \& Vie, 1137, 52-53, 56-57, 66.

[7] Cohen-Tannoudji, G. and Spiro, M. (2013) Le boson et le chapeau mexicain. Gallimard, Folio Essais, 268-275, 318, 423.

[8] Bagdoo, R. (2011) Cosmological Inconstant, Supernovæ 1a and Decelerating Expansion. http://vixra.org/abs/1304.0169 https://www.academia.edu/5539777

[9] Bagdoo, R. (2016) Neutrino's Temporal Oscillations. 7, 11, 15. http://vixra.org/abs/1605.0005 https://www.academia.edu/25111027

[10] Klein, É. (2007) Le facteur ne sonne jamais deux fois. Éditions Flammarion, Paris, 239.

[11] Magnan, C. (2011) Le théorème du jardin. amds édition, 203.

[12] Hotson, D.L. (2002) Dirac's Equation and the Sea of Negative Energy. Part 1, Infinite Energy, Issue 43.

[13] Zitoun, R. (1998) La physique des particules. Nathan Université, 32-34.

[14] Michaud, A. (2017) Electromagnetic Mechanics of Elementary Particles. Scholars' Press, 2nd Edition, 297-326, 329, 537.

[15] Pharabod, J.-P. and Pire, B. (1993) Le rêve des physiciens. Edition Odile Jacob.

[16] Bagdoo, R. (2008) The Pioneer Effect: A New Theory with a New Principle. http://vixra.org/abs/0812.0005 https://www.academia.edu/5535864/

[17] Bagdoo, R. (2013) The Energy in Virtue of the Principle of Compensation. http://vixra.org/abs/1301.0180 https://www.academia.edu/5539802/

[18] Bagdoo, R. (2017) The Equation of The Universe. http://vixra.org/abs/1709.0033 https://www.academia.edu/34546347/ 